\title{
The Aetiopathogenesis of Late Inflammatory Reactions (LIRs) After Soft Tissue Filler Use: A Systematic Review of the Literature
}

\author{
Y. Bachour' ${ }^{1}$ (D) J. A. Kadouch ${ }^{2}$ F. B. Niessen ${ }^{1}$
}

Received: 26 August 2020/ Accepted: 11 April 2021/Published online: 28 April 2021

(C) The Author(s) 2021

\begin{abstract}
Background Late inflammatory reactions (LIRs) are the most challenging complications after filler use. The immune system plays a prominent role in its etiology, albeit to an unknown extent. Bacterial contamination in situ has been hypothesized to be causative for LIRs. How this relates to the immunological processes involved is unknown. This article aims to provide an overview of immunological and bacterial factors involved in development of LIRs.

Methods We undertook a systematic literature review focused on immunological factors and microbiota in relation to LIRs after filler use. This systematic review was performed in accordance with the PRISMA guidelines. PubMed, EMBASE and the Cochrane databases were searched from inception up to August 2019. Included studies were assessed for the following variables: subject characteristics, number of patients, primary indication for filler injection, implant type/amount and injection site, type of complication, follow-up or injection duration, study methods, type of antibiotics or medical therapies and outcomes related to microbiota and immunological factors.
\end{abstract}

Supplementary Information The online version contains supplementary material available at https://doi.org/10.1007/s00266021-02306-3.

Y. Bachour

yarabachour@gmail.com

1 Department of Plastic, Reconstructive and Hand Surgery, Amsterdam UMC-location VUmc, De Boelelaan 1117, PO Box 7057, 1007 MB Amsterdam, The Netherlands

2 Department of Dermatology, ReSculpt Clinic, Amsterdam, The Netherlands
Results Data on immunological factors and bacterial contamination were retrieved from 21 included studies. Notably, the presence of histocytes, giant cells and Staphylococcus epidermidis within biopsies were often associated with LIRs.

Conclusion This review provides a clear overview of the immunological factors associated with LIRs and provides a hypothetical immunological model for development of the disease. Furthermore, an overview of bacterial contamination and associations with LIRs has been provided. Follow-up research may result in clinical recommendations to prevent LIRs.

Level of Evidence III This journal requires that authors assign a level of evidence to each article. For a full description of these Evidence-Based Medicine ratings, please refer to the Table of Contents or the online Instructions to Authors-www.springer.com/00266..

Keywords Filler - Soft tissue filler - Dermal filler . Complications - Adverse events · Late inflammatory reactions - Aetiopathogenesis

\section{Introduction}

Fillers, also referred to as dermal fillers, soft tissue fillers or dermal implants have been used for decades, mainly for cosmetic reasons, but also to improve aesthetic outcome after trauma, cancer or malformations [1]. They are bioinjectable materials that are approved by the FDA as medical devices or implants. Soft tissue fillers are injected transcutaneously through a needle or cannula into/or between the dermis and subcutaneous fat. It is a noninvasive surgical procedure that must be performed in a setting where preoperative antiseptic requirements are taken into 
account [2]. Over the past decade, their use for aesthetic purposes has increased exponentially. With more than 2.5 million procedures in 2018, it has been the most frequent non-surgical procedure in plastic surgery in the USA [3]. The increase of their use has subsequently resulted in an increase of soft tissue filler products. Although there is no universally accepted classification for these products, they are mostly classified by their biodegradability into temporary-, biostimulatory- or permanent fillers $[4,5]$. Biostimulatory fillers (formerly referred to as semi-permanent) exert their definitive filling effect indirectly by inducing volumization through neocollagenesis stimulation at the site of injection [4]. An overview of the most used (in present and past) soft tissue fillers according to this classification can be found in Table 1 .

Although manufacturers and several studies claim that adverse events after dermal fillers are very uncommon, unwanted adverse events do occur with all fillers products (Fig. 1a, b) [6-12]. A review by de Vries et al. shows more specifically that adverse events have been reported for almost every fillers product [13]. Of these, late filler reactions such as lumps, nodules, swellings or granulomas are the most challenging to treat since their exact aetiopathology is unknown [7]. Inflammatory nodules usually emerge between several weeks up to several years after the procedure and are therefore also called late inflammatory reactions (LIRs) [14]. It is estimated that these complications occur in $0.01 \%-0.1 \%$ of the procedures that have been carried out [15]. Moreover, especially the permanent filler-induced LIRs, often cause a permanent disfigurement of the faces of treated patients [4, 9, 16, 17].

A foreign body reaction is a natural tissue response after soft tissue filler injection [18]. The immune response around the filler material involves macrophages, histiocytes, lymphocytes and giant cells $[10,12,19]$. In time, these cells reduce in numbers and chronic inflammation cells accumulate around and within the filler material [5].
An inflammatory nodule may then be formed [20]. To date, the aetiopathology of LIRs is unknown and there is much debate about the pathogenesis of this disorder. Many authors have focused on injection techniques and types of filler products in relation to the development of LIRs [21, 22]. However, adaptation of filler characteristics has not significantly diminished the incidence of LIRs. Several risk factors have been identified, and additional causative factors have been proposed, such as occurrence of a subclinical infection, an excessive foreign body reaction and biodegradability of the filler material $[15,18,23,24]$. Although none of these theories are conclusive so far, there is consensus that the immune system plays a prominent role in the development of LIRs, albeit to an unknown extent [25-27].

Bacterial contamination of the filler material has frequently been proposed as a likely cause of LIRs and some indications for this theory exist $[7,8,28]$. To understand the possible role of contamination, a clear picture of the immunopathogenesis of LIRs is necessary. Therefore, the aim of this systematic review is twofold: (1) to give an overview of immunological factors involved in LIRs, and (2) to determine the role of bacterial contamination in LIRs. Although nowadays most permanent fillers are banned for cosmetic use, complications following their injection in the past are still seen in current clinical practice. Therefore, we have chosen to include them the literature search and review for this article.

\section{Materials and Methods}

\section{Literature Search}

A systematic review was performed of the literature pertaining to immunological factors and bacterial contamination/infection of LIRs after soft tissue filler use. This

Table 1 Overview of dermal implants

\begin{tabular}{llll}
\hline Biodegradability/longevity & Substances & Brand names & $\begin{array}{l}\text { Duration of } \\
\text { effect }\end{array}$ \\
\hline $\begin{array}{l}\text { Temporary } \\
\text { Biostimulatory }\end{array}$ & $\begin{array}{l}\text { Collagen (not used anymore), hyaluronic acid } \\
\text { Polylactic-L-Acid (PLA), calcium hydroxylapatite } \\
\text { (CHA), polycaprolactone }\end{array}$ & $\begin{array}{l}\text { Restylane, Juvéderm, Belotero } \\
\text { Radiesse, Sculptra Ellansé }\end{array}$ \\
Permanent & $\begin{array}{l}\text { Silicone, polyalkylimide gel (PAIG, Bio-Alcamid), } \\
\text { polyacrylamide gel (PAAG, Aquamid), polymethyl- } \\
\text { methacrylate (PMMA, Artocoll/ArteFill), HEMA/ }\end{array}$ & $\begin{array}{c}\text { Artefill, Dermalive, Aquamid, Bio- } \\
\text { Alcamid }\end{array}$ & \\
& EMA (DermaLive) & & 12-36 months \\
\hline
\end{tabular}

An overview of the different dermal implants according their biodegradability. This concerns the longevity of the dermal implant after injection and varies from temporary, to biostimulatory and permanent implant 


\section{(a)}

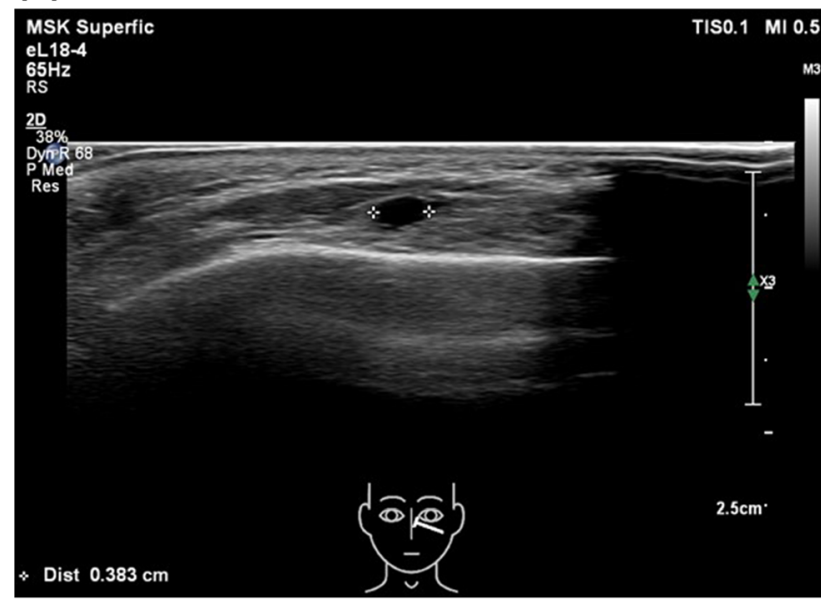

(b)

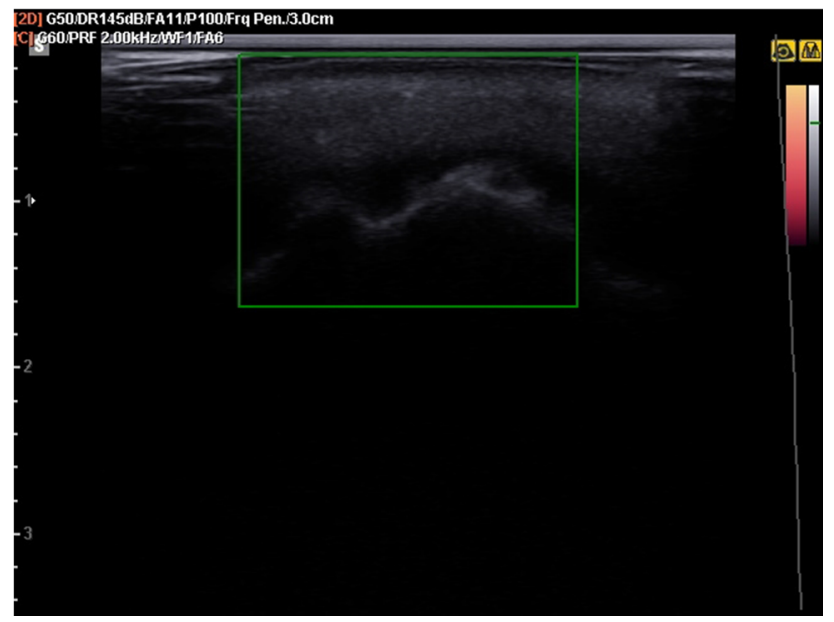

Fig. 1 Ultrasound images of a filler depots. a On this ultrasound image of the tear trough, a $40 \mathrm{~mm}$ sized anechoic (black) process is seen, which matches the image of hyaluronic acid filler. b On this ultrasound image of the cheek area of a patient injected with a calcium hydroxylapatite filler. We see a cloudy isoechoic cloudy depot with multiple hyperechoic dots (=calcium hydroxylapatite particles), preventing ultrasound penetration good definition of underlying anatomical structures

systematic review was carried out in accordance with the Preferred Reporting Items for Systematic Reviews and Meta-Analyses Statement (PRISMA) guidelines [29]. An extensive search was conducted using the electronic databases PubMed, Embase.com and Wiley/Cochrane Library. Appropriate keywords in the English language were combined by Boolean logical operators and adapted to the appropriate syntax of each database. The following terms were used (including synonyms and closely related words) as index terms or free-text words: 'adverse events' and 'filler.' The full search strategies for all the databases can be found in the Supplementary Information. Studies written in English and Dutch from September 1975 until August 2019 were reviewed.

\section{Selection Criteria}

We searched for original studies on LIRs after soft tissue filler use which reported on microbiota and/or immunological factors. All patient studies as well as relevant animal studies were included. Of these studies, all types of filler products were included, as well as all sites of injection. In vitro studies and studies which investigated the normal immune response of filler products were excluded. Case reports less than 5 patients and isolated abstracts were excluded as well as non-original studies such as reviews, editorials, communications, correspondence, discussions and letters.

The search was executed in cooperation with a medical information specialist. The article selection was performed by two reviewers in two steps: in the first step eligibility of the articles was screened based on the title. Subsequently, the abstracts of the selected articles were evaluated, and, in case of doubt, the full article was reviewed. Articles which met the inclusion criteria were included in the systematic review.

\section{Data Collection and Analyses}

Data were extracted using standardized tables developed for this purpose. Data included the following variables: authors, year of publication, study design, number of patients or samples, type/amount and location sites of injected filler, included group of subjects, primary indication for injection, type of complication, follow-up or injection duration, microbial/immunological methodologies, type of antibiotics/medical therapies, main outcomes and the conclusion. Included studies were assigned a level of evidence according to the Oxford Centre for EvidenceBased Medicine [30].

Due to heterogeneity of the studies, statistical metaanalysis of the data is impossible. Instead, we performed qualitative and descriptive analyses of the outcomes.

\section{Results}

\section{Article Demographics}

The literature search produced 2340 articles after removal of duplicates. Of these, 487 remained after title screening, and 197 after evaluation of the abstracts. Full text review yielded 21 studies which met the inclusion criteria (Fig. 2). Studies were classified into two major subject groups: (1) immunological factors and (2) bacterial contamination. The baseline characteristics of included studies are given in Table 2. 
Fig. 2 Study selection flowchart

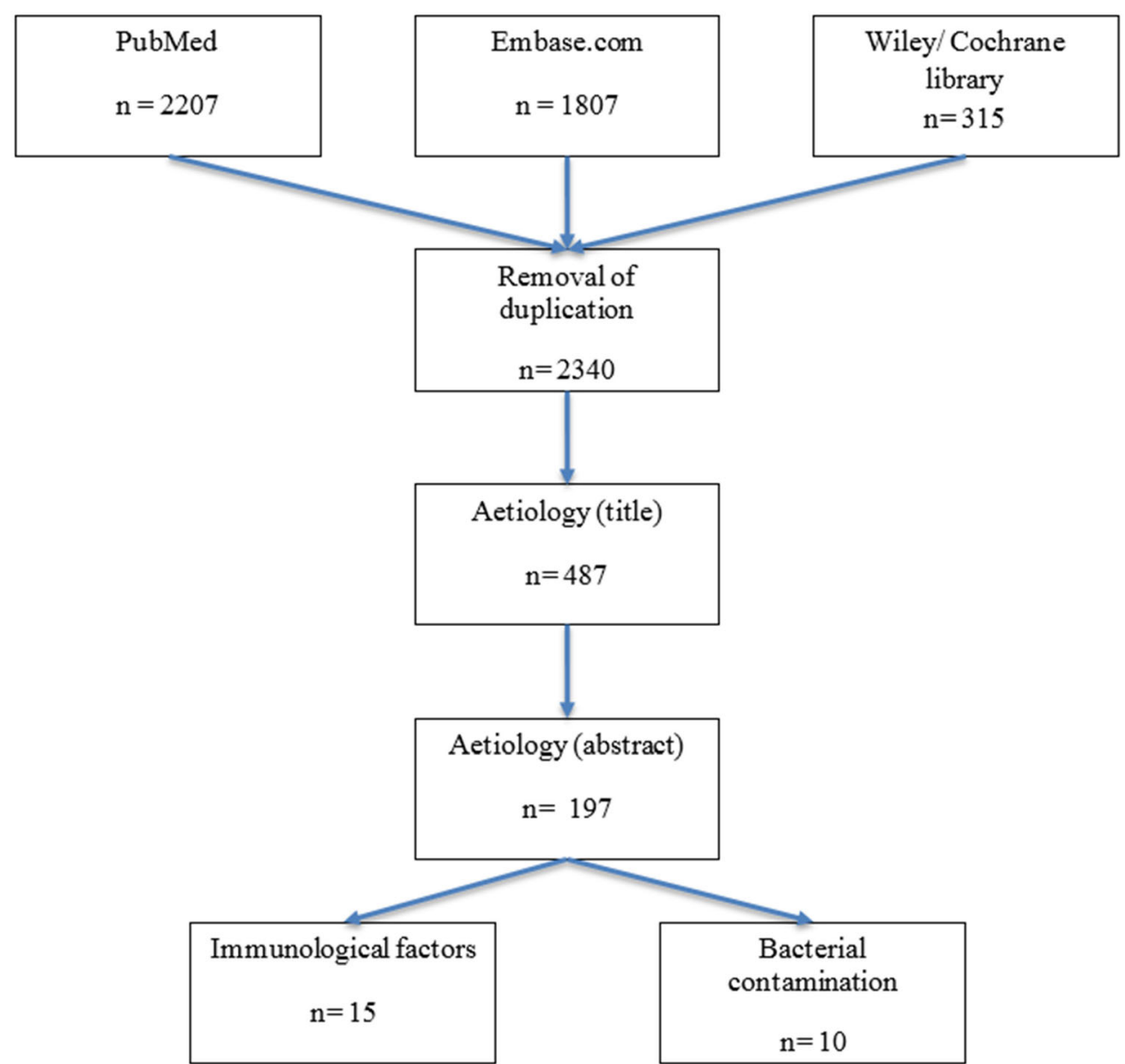

\section{Bacterial Contamination}

Eight human studies (including 168 patients) have investigated the presence of bacteria on samples from LIRs after filler use [7, 8, 19, 23, 28, 31-33]. Six studies were crosssectional studies, while two studies were case series. Furthermore, two human study (including 30 patients) and one animal study investigated the effect of systemic antibiotics for the treatment of LIRs to prove the role of bacterial contamination in the aetiopathogenesis of filler-related LIRs. These were a cross-sectional study and a case series.

\section{Immunological Factors}

We found 14 studies with regard to a variety of immunological factors in relation to LIRs $[1,5,10-12,18,19,25-27,32-35]$. These studies with a total of 295 patients described local presence of immune cells or immunological factors using histological or histochemical techniques. Eleven studies were case series, two were cross-sectional studies, while one study was a casecontrol study. Systemic immunological factors were reported in 2 studies including 15 patients in total. Two studies reported on the systemic as well as on the local presence of immune cells. Several groups investigated whether local and/or systemic immunological factors were associated with adverse events, by investigating the role of medical therapies. Two human studies (one cross-sectional study and one case-control study) and one animal study used medical therapies for preventing or diminishing LIRs.

\section{Discussion}

\section{Bacterial Contamination and LIRs}

The exact aetiopathology of LIRs caused by filler is still poorly understood. Several authors have suggested that delayed-onset complications can occur in response to local or systemic infections, (facial) injuries, systemic medication or vaccinations, or invasive treatments (such as dental surgery) in the vicinity of the filler deposits [8, 36-44].

Numerous human studies have shown the presence of bacteria in material derived from patients with LIRs (Table 7 in ESM) [7, 8, 23, 28, 31, 32]. Two studies did not detect any bacteria in the collected samples $[19,33]$. The 


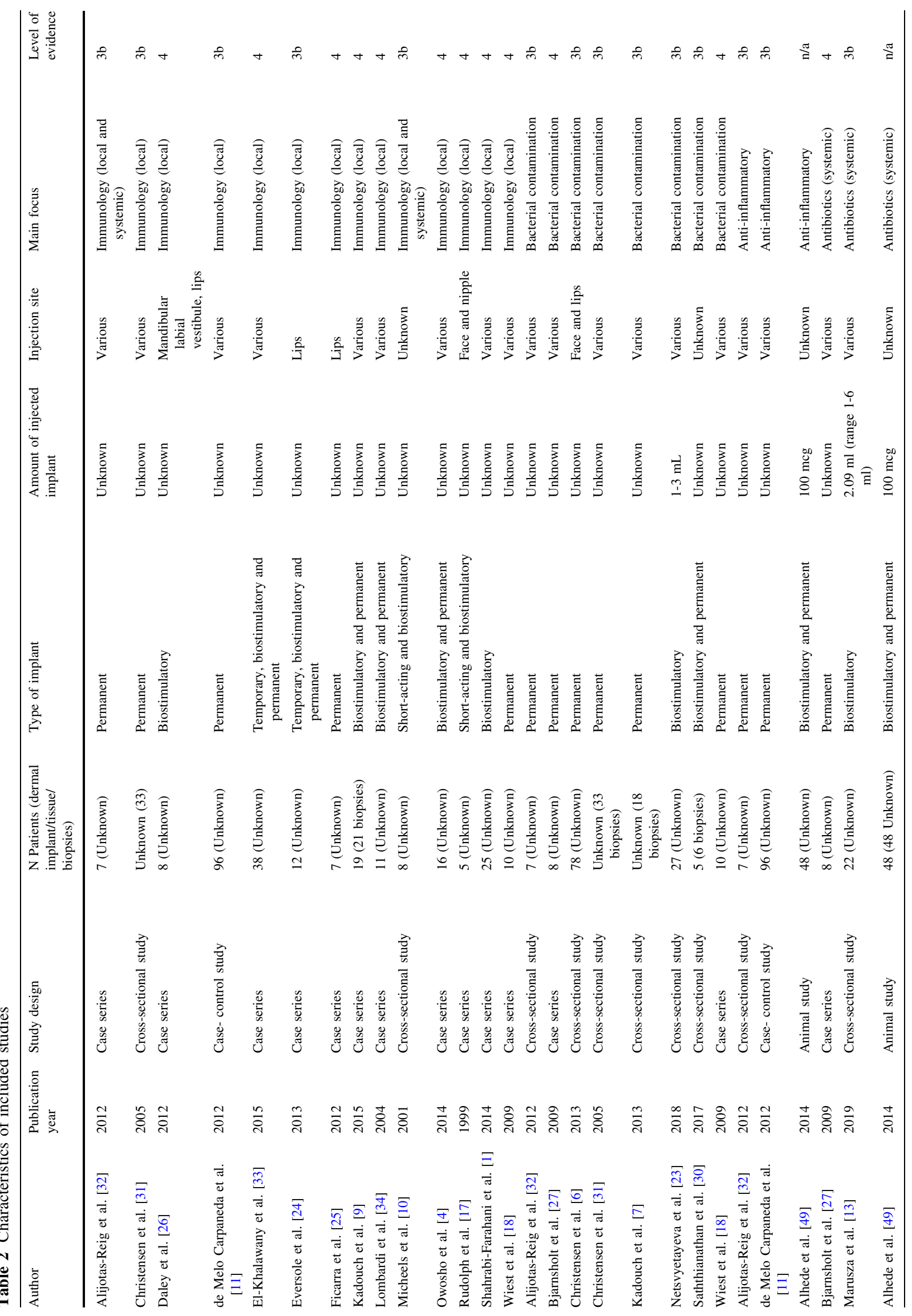


most common detected bacteria were Staphylococcus spp. (mainly Staphylococcus epidermidis) and Propionibacterium acnes, which were detected in up to $98 \%$ of positive cultures, and which lead to the hypothesis that the presence of these bacteria is involved in development of LIRs [7, 8, 24, 28, 31, 32]. Two studies compared bacterial presence in samples from patients with adverse events and patient with no adverse events, both finding a much higher rate of bacteria in the adverse events' group [7, 24].

In a human study by Christensen et al., they showed that bacterial presence varies among different filler compounds, with an increased contamination within the permanent filler material [32]. This finding has been supported by one animal study by Alhede et al. This experiment left contaminated gels in a mouse model finding a sustainment of bacterial growth within the permanent gel, less in the biostimulatory gel and no growth within the temporary gel [45]. The latter might explain the difference in complication rate between permanent, biostimulatory and temporary filler material.

Despite this evidence for the presence of bacteria around filler material and support for a primary role in the development of LIRs, some authors postulate that these findings are not enough to conclude LIRs have an infectious aetiopathology [46]. They state that the proof of such a conclusion cannot be based on the visualization of a few bacteria on histologic slides or by genetically identifying them, but to demonstrate the presence of a biofilm formation with hundreds of bacteria.

\section{Immunological Factors Associated with LIRs}

Multiple studies indicate that extrinsic factors such as (micro-) trauma, infections and drugs or vaccinations seem to be capable of activating certain mechanisms leading to delayed-onset complications such as (low-grade) inflammatory nodules and abscess formation (Fig. 3). Although the exact underlying mechanisms responsible for eliciting such complications remain unknown, filler characteristics and host immune status both appear to play a role [8, 37, 47-50]. In our literature search, we found that several studies have evaluated the role of immunological factors in the development of LIRs and show involvement of several cell types inducing inflammatory processes (Figure 3, Table 6 in ESM) [1, 5, 10-12, 18, 19, 25-27, 32-35]. Namely (epithelioid) macrophages, histiocytes, lymphocytes, giant cells were demonstrated HE stained sections of these filler nodules. In some cases also, neutrophils, eosinophils or (plasmacytoid) dendritic cells (DCs) were found.

\section{Foreign Body Reactions}

The generation of a granulomatous foreign body reaction (GFBR) is a 'normal,' physiological response from the host to any foreign body. All filler agents used for soft tissue augmentation are thought to elicit some degree of granulomatous inflammatory reaction following injection $[49,51]$. To a certain point, this is considered to be part of a normal physiological response [49, 52]. GFBR can be classified according to a severity grading system and/or into different clinicohistological phenotypes. Duranti proposed a 4-point grading system for the severity of GFBR [20]. Lemperle and Lombardi proposed a classification of GFBR into different clinical and/or histological subtypes [35, 49]. A genuine GFBR is predominantly composed of histiocytes/macrophages and giant cells encapsulating filler particles [20, 49]. The exact pathophysiology of filler-induced GFBR, or 'filler granulomas,' has yet to be elucidated.

Current insights differentiate several steps in host reaction on biomaterial implantation. Within minutes after implantation, host plasma components such as albumin, fibrinogen, fibronectin, lipids, sugars, ions and platelets are absorbed on the surface of the implant [7, 22, 53]. Platelets and other components of the coagulation cascade induce clot formation, which also functions as the provisional matrix around the biomaterial. Platelet adhesion and activation, and the release of pro-inflammatory cytokines, chemokines and growth factors induce sequentially the acute and chronic inflammatory responses on the implanted material. Damage-associated molecular patterns (DAMPs), pathogen-associated molecular patterns (PAMPs) and Alarmins present at the implantation site activate macrophages, leukocytes and dendritic cells through pattern recognition receptors (PRRs), toll-like receptors (TLRs) and C-type lectin [45-47, 53-55]. In the acute phase, the recruited neutrophils attempt to degrade the biomaterial through phagocytosis and the release of proteolytic enzymes, reactive oxygen species (ROS) and neutrophil extracellular traps (NETs, consisting of granular proteins, elastase, histones and chromatin DNA) [50, 51, 53]. NETs are usually involved in trapping pathogens and prevention of infection spread.

The release of immune-regulatory signals by neutrophils attracts monocytes, macrophages, immature DCs and lymphocytes, promotes monocytes differentiation into 'M1'-type macrophages secreting pro-inflammatory cytokines (Interleukin (IL-) 1 $\beta$, IL-6, necrosis factor alpha $(\mathrm{TNF}-\alpha)$ ) and downregulates the presence and activity of neutrophils themselves. The next step in the healing process is a shift toward 'M2'-type macrophages secreting anti-inflammatory cytokines (i.e., IL-10) and recruiting fibroblasts for effective tissue regeneration. The shift 


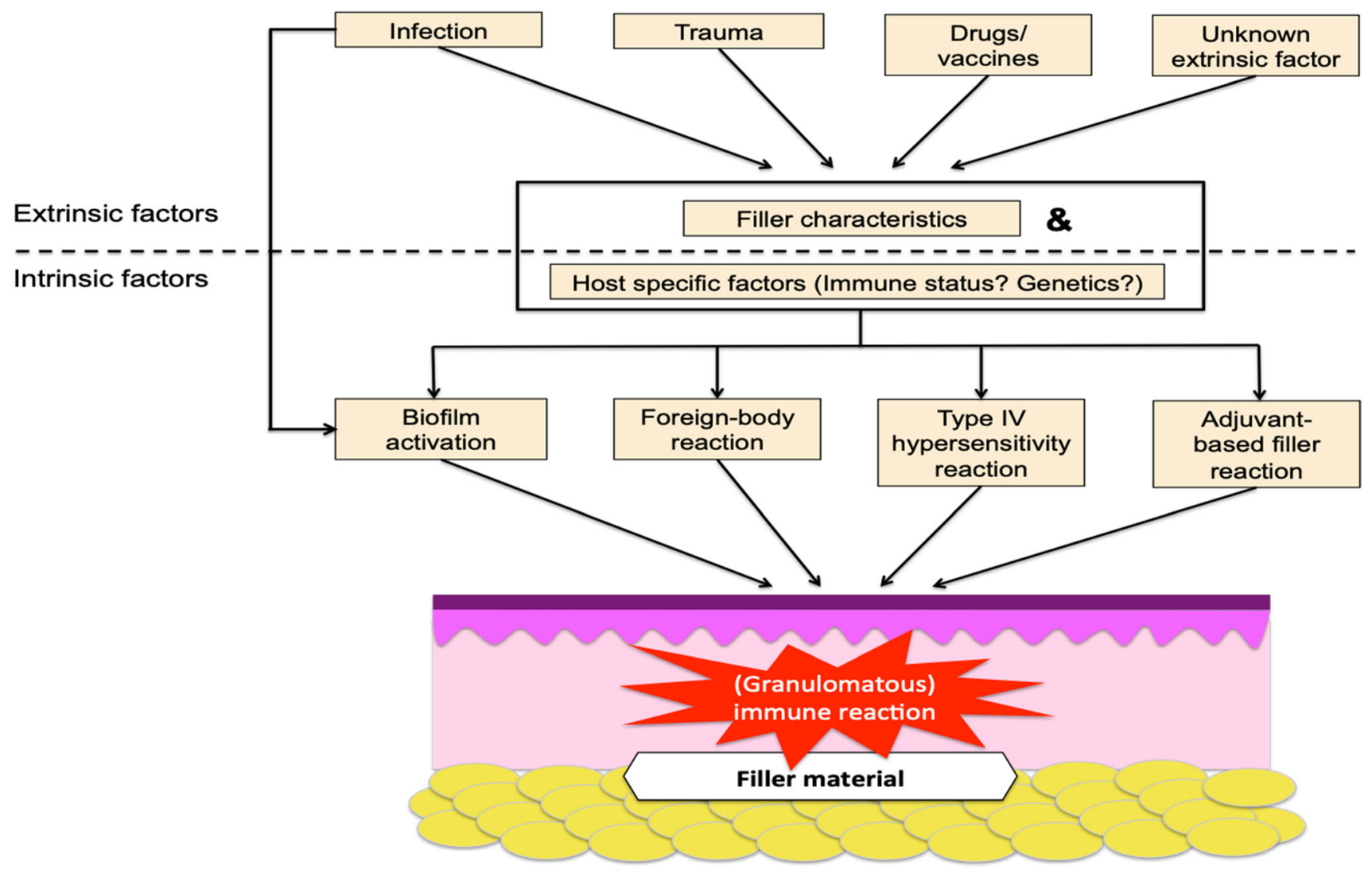

Fig. 3 Extrinsic and intrinsic factors in the development of delayedonset complications of fillers Late inflammatory reactions of injected filler usually considered to be a result of foreign body reactions, microbial contamination (in biofilms or otherwise) of filler material, type IV hypersensitivity reactions or adjuvant-based filler reactions.

toward an anti-inflammatory 'M2'-type healing response can be altered by specific characteristics of the implanted material. For example, particles larger than $5 \mu \mathrm{m}$ require the presence of aggregated macrophages, or giant cells, to be phagocytosed [56-58]. Particles larger than 15 to $20 \mu \mathrm{m}$ are generally not subject to ingestion by macrophages by true phagocytosis, but may be enclosed by giant cells. Failure of effective phagocytosis leads to a chronic inflammation pathway and granuloma formation, consisting of macrophages and giant cells, as well as a contiguous infiltrate of lymphocytes secreting pro-inflammatory cytokines (i.e., tumor necrosis factor alpha (TNF- $\alpha$ ), interferon gamma (IFN- $\gamma$ ) and interleukin 12 (IL-12)) [43, 53]. Similarly, excessive production of NETs by neutrophils can impair healing and lead to a chronic inflammation and encapsulation [53, 57, 58].

Numerous studies have been performed on the different structural properties of filler, such as chemical composition, electrical charge, surface irregularities or particle size and the presence of contaminants, which are of known influence on host immune responses [49, 50] (Fig. 3, Table 3).
Although the underlying pathomechanisms are largely unresolved, they are probably influenced by host specific factors, such as immune status and genetic background, as well as by characteristic of the filler material itself

The relation with time can also be of importance. Most studies show a decreased inflammatory reaction after several months; however, this also depends on the type of filler used [32]. Whereas material from acute lesions (up to 30 days) shows a cellular infiltrate composed mainly of neutrophils, lymphocytes, macrophages and cells similar to fibroblasts, tissues from 6 months on show predominantly the presence of small and round empty spaces surrounded by dense and organized inflammatory and fibrous tissue. In some cases, capsule formation was reported.

\section{Delayed-Type Hypersensitivity Reactions}

Immune-mediated hypersensitivity to fillers in patients with LIRs has been investigated by one study. Micheels et al. showed that skin tests in patients with adverse events were positive for one or the other or both of the injectable hyaluronic acid preparations [11]. Also, serum analysis revealed positive antibodies against Restylane and/or Hylaform and even IgG and E anti-hyaluronic acid. Delayed hypersensitivity reactions are often proposed in the literature as cause for LIRs. Some authors have in fact 
postulated that all granulomatous reactions to fillers are in fact type IV (delayed-type) hypersensitivity reactions (Fig. 3) [59]. However, it must be noted that a true type IV hypersensitivity reaction is a systemic immune response that should affect all injected sites at the same time [23, 60]. In addition, to our knowledge non-autologous (bovine) collagen and hyaluronic acid are the only two main filler constituents reported to be capable of eliciting type IV hypersensitivity reactions, substantiated by positive skin-testing $[11,17,61,62]$. Bovine collagen is the carrier for PMMA fillers, whereas hyaluronic acid has by far been the most widely used temporary filler this past decade. Although, T cell-mediated delayed-type hypersensitivity reactions to these constituents could play a role in LIRs to these two filler types, have not found any literature, other than the paper by Micheels et al., supporting this hypothesis with golden standard allergic testing.

\section{Adjuvant-Based Filler Reactions}

Alijotas-Reig recently postulated that fillers may act as adjuvants, rather than as antigens [37]. Adjuvants are defined as substances that may stimulate immune responses without having specific antigenic properties themselves [63]. Both the innate and adaptive immune systems are influenced by the effects of adjuvants. Adjuvants enhance innate immune responses by mimicking evolutionary conserved molecules (e.g., PAMPs) capable of binding tolllike receptors (TLRs, mainly activating TLRs 1, 4, 5, 7 and 9 ), resulting in the release of $T_{h} 1$ inflammatory cytokines [63]. They also increase the activity of dendritic cells
(DCs), lymphocytes and tissue macrophages. The immuneenhancing effects of adjuvants are supposedly mediated by five different activities, as depicted in Table 4 [63, 64]. Certain triggers such as infection, trauma and vaccination may induce adjuvant activity or act as adjuvants themselves [37, 63-66]. In addition, sequential exposure to different adjuvant stimuli is believed to increase the risk of abnormal immune responses [37, 66]. Several studies on animals and humans have demonstrated that adjuvants are able to induce autoimmunity and autoimmune diseases [63]. Recently, the name 'autoimmune/inflammatory syndrome induced by adjuvants,' in short 'ASIA' or Schoenfeld's syndrome, was introduced to describe the spectrum of immune-mediated systemic diseases that may be triggered by previous exposure to an adjuvant stimulus [67, 68] (Table 5). Several studies have demonstrated similar systemic inflammatory responses after the use of filler [37, 47, 48, 59, 65, 69-71]. Yet unknown factors, related to the specific adjuvant(s) involved and to the extent in which innate, adaptive and regulatory immune responses are activated, are believed to determine whether an autoimmune response remains limited or will evolve into full-blown systemic disease [37, 47, 48]. Genetic predisposition for the development of ASIA is also suspected $[37,47,66]$. Known adjuvants that may cause ASIA are silicone, aluminum salts, pristane and infectious components [63, 65, 67]. In addition, hyaluronic acid compounds and acrylamides have also been identified as adjuvants [16, 37, 47, 48, 71, 72]. More researched is needed to support and further establish this hypothesis.

Table 3 Chemical properties of dermal implants and their immunological effect [56]

\begin{tabular}{|c|c|}
\hline Structural properties & Immunological effect \\
\hline \multicolumn{2}{|l|}{ Electrical charge } \\
\hline Positively charged particles & Attract and/or activate macrophages \\
\hline Negatively charged particles & Repel some negative charged bacteria \\
\hline \multicolumn{2}{|l|}{ Surface irregularities } \\
\hline Irregular surfaces & Elicit a longer-lasting inflammatory reaction \\
\hline Smooth surfaces & Formation of a thicker fibrous capsule around the material \\
\hline \multicolumn{2}{|l|}{ Particle size } \\
\hline Large particles $(>20 \mu \mathrm{m})$ & No phagocytosis \\
\hline Small particles $(<20 \mu \mathrm{m})$ & Fast phagocytosis, resulting in a greater local inflammatory reaction \\
\hline \multicolumn{2}{|l|}{ Hydrophilic/hydrophobic } \\
\hline Hydrophilic polymer gels & Highly biocompatible and easily penetrable by nutrients and waste products \\
\hline Hydrophobic polymer gels & $\begin{array}{l}\text { Favor fibronectin absorption and therefore cellular adhesion, thus promoting } \\
\text { a pro-inflammatory response }\end{array}$ \\
\hline \multicolumn{2}{|c|}{ HA implants (molecular size and the amount of chemical cross-linking) } \\
\hline Low molecular weight HA & Acts pro-inflammatory and triggers the immune system \\
\hline High molecular weight HA & Acts anti-inflammatory properties \\
\hline
\end{tabular}




\section{Medical Therapies}

Anti-inflammatory medications have been investigated in several studies as possible therapy for LIRs (Table 8a in ESM) [12, 14, 28, 33, 45]. Although some studies are promising, contradictive results exist [12, 14, 28, 33, 45]. Tacrolimus has been used as an immunosuppressant drug in transplantations and granulomatous disease [73]. Alijotas-Reig et al. investigated its use in series of seven patients with late-onset adverse events after silicone injection, finding good clinical response [33]. De Melo Carpaneda et al. investigated in a series of seven patients the effect of intralesional corticosteroids [12]. These patients reported an initial recovery with softening of the compromised region, but after a few months, the nodule became harder and the skin of the region where the product had been injected turned whitish and depressed indicating corticosteroid induced atrophy. Alhede et al. investigated in their animal model the effect of triamcinolone acetonide in combination with antibiotics [45]. They contaminated gels and left them for seven days in a mouse model. This study showed that once the bacteria had settled (into biofilms) within the gels, even successive treatments with high concentrations of relevant antibiotics/corticosteroids were not effective.

Also an antimicrobial approach for the treatment of LIRs has been widely addressed (Table 8b in ESM). The hypothesis stating bacteria in situ are involved in the development of adverse events is further investigated by Marusza et al. who treated patients with an antibiotic scheme of whom all experienced resolution of symptoms and with no recurrence of their complication [14]. However, these results must be interpreted with care, as the used antibiotic (clarithromycin) is also known for its immune modulating properties and the used treatment scheme also included intralesional hyaluronidase injections which dissolves the filler. Therefore, it is not possible to draw conclusions based on this study.

Although a lot has been written on the best treatment approach for LIRs, to date no evidence-based effective therapy for LIRs exists. To achieve this, first the aetiopathological uncertainties most be solved, keeping in mind that maybe not all LIRs have the same aetiopathology or cause. Also, although bacterial presence might not necessarily prove an infectious process, bacterial contamination of the filler material might act as stimulus for other (innate) immune response. This is why many practitioners use and will keep using immune modulating antibiotics as first line therapy in case of HA-induced LIRs. This way treats both an infectious and an inflammatory reaction.

The best approach, however, remains prevention. For this reason, possible causes or triggers for an infectious or inflammatory process should be avoided. Filler treatments should therefore be performed in under aseptic conditions by well-trained physicians with knowledge of skin preparation, facial anatomy and potential complications. Like described by De Boulle and Heydenrych, special considerations are necessary for patients with preexisting autoimmune or -inflammatory diseases [17].

It must also be empathized that (chemical properties of) permanent fillers have been proven to have a significantly higher risk for adverse events such as bacterial contamination, inflammatory reactions, abscesses and migration up to decades after injection. Their use should therefore be discouraged, especially for aesthetic indications.

\section{Recommendations for Injection Techniques and Treatment Options}

As previously mentioned, dermal fillers can be used for cosmetic but also for medical reasons. For both indications, there might be a loss of elasticity and collagen in the dermis, volume loss of the subcutaneous fat and bone

Table 4 Adjuvant immunological effect exerted by different modes of action [63]

\begin{tabular}{|c|c|c|}
\hline No. & Mode of action & Immunological effect \\
\hline 1. & $\begin{array}{l}\text { Translocation of antigens to the lymph nodes where they can } \\
\text { be recognized by T cells }\end{array}$ & $\begin{array}{l}\text { Enhancing } \mathrm{T} \text { cell activity, increased clearance of pathogen throughout the } \\
\text { organism }\end{array}$ \\
\hline 2. & $\begin{array}{l}\text { Protection of antigens, which grants the antigen a prolonged } \\
\text { delivery and exposure }\end{array}$ & $\begin{array}{l}\text { Upregulating the production of } \mathrm{B} \text { and } \mathrm{T} \text { cells needed for enhanced } \\
\text { immunological memory as part of the adaptive immune response }\end{array}$ \\
\hline 3. & $\begin{array}{l}\text { Increasing the capacity to cause local reactions at the } \\
\text { injection site }\end{array}$ & $\begin{array}{l}\text { Greater release of danger signals by chemokine releasing cells such as } \mathrm{T} \\
\text { cells and mast cells }\end{array}$ \\
\hline 4. & Inducing the release of inflammatory cytokines & $\begin{array}{l}\text { Recruitment of } \mathrm{B} \text { and } \mathrm{T} \text { cells at sites of infection and increased } \\
\text { transcriptional events leading to a net increase of immune cells as a whole }\end{array}$ \\
\hline 5. & $\begin{array}{l}\text { Interacting with pattern recognition receptors (PRRs), } \\
\text { specifically toll-like receptors (TLRs), on accessory cells }\end{array}$ & Increase of the innate immune response to antigen \\
\hline
\end{tabular}


Table 5 Criteria for the diagnosis of ASIA* as suggested by the group of Dr. Shoenfeld [63]

\section{Major criteria}

Exposure to an external stimulus (infection, vaccine, silicone, adjuvant) prior to clinical manifestation of symptoms

The appearance of 'typical' clinical manifestations:

Myalgia, myositis or muscle weakness

Arthralgia and/or arthritis

Chronic fatigue, unrefreshing sleep or sleep disturbances

Neurological manifestations

Cognitive impairment, memory loss

Pyrexia, dry mouth

Removal of an inciting agent induces improvement

Typical biopsy of involved organs

Minor criteria

The appearance of autoantibodies or antibodies directed at the suspected adjuvant

Other clinical manifestations (i.e., irritable bowel syndrome)

Specific HLA haplotypes (i.e., HLA DRB1, HLA DBQ1)

Evolvement of an autoimmune disease (i.e., MS, SSc)

structures. As a result of this, injection of fillers needs a three-dimensional approach. The injection of fillers is therefore divided into three injection levels: deep dermal, subdermal and supraperiosteal [74]. Although there is not a clear algorithm for the injection of fillers in the tissue, there is a consensus for the following injection techniques for different types of fillers; HA skinboosters should be injected into the dermis using a thin needle. Biostimulatory and HA fillers could be injected subdermal with the use of a cannula using the fanning technique. However, biostimulatory fillers should not be used in the periorbital and perioral area as there are muscular dynamics that cause a movement of the injected filler. Biostimulatory and HA fillers can be injected on the periost using a needle. Here, it is advised to inject low volume of the compound (around $0,025 \mathrm{~mL}$, the so called 'JVL bolus') in order to prevent any vascular adverse events.

Based on this review, we propose the following treatment algorithm for LIRs (Fig. 4). As stated above, culture proven infections are common after filler injection. Therefore, when clinical features of inflammation such as oedema, heat, erythema, tenderness or pain are present, the first choice should be conservative treatment with oral antibiotics. We recommend the use of tetracyclines because of their dual action as antimicrobial and ant-inflammatory medication. Examples are doxycycline or minocycline (100-200 mg daily). In some cases, nonsteroidal anti-inflammatory drugs (NSAIDs) or oral corticosteroids can be added. When conservative treatment fails surgical treatment is required.

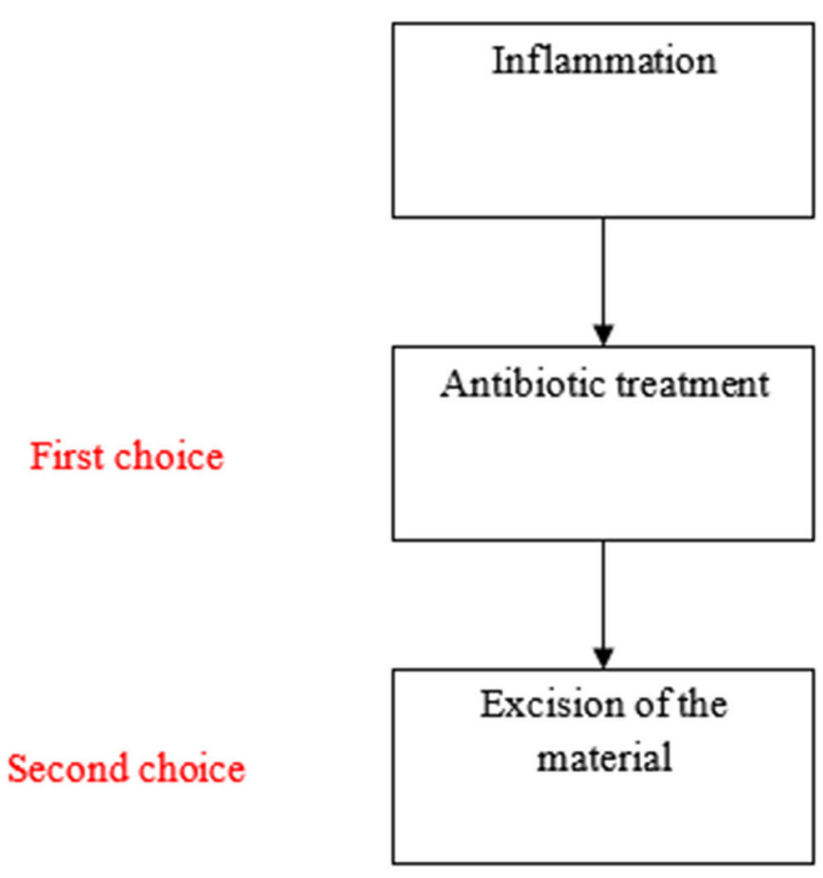

Fig. 4 Algorithm for treatment of LIRs

\section{Conclusion}

This review has analyzed the aetiopathological hypotheses of LIRs that can currently be found in the literature. A major role is seen for a (pathological) foreign body reaction led by activated histocytes and giant cells, eventually resulting in a chronic inflammation. S. epidermidis is the bacterium most often found in LIRs although its role is still 
debated (contamination and infection, or merely an immunological trigger?). Several substances, such as antibiotics and corticosteroids, seem to be effective in the treatment of LIRs, although the mechanism is not fully understood, and more research is needed.

Funding This study is funded by the Netherlands Organisation for Scientific Research and Development (ZonMw), grant number 516001352. ZonMw did not have any involvement in the study design, data collection, analysis or interpretation, nor in writing the report and decision to submit for publication. No funding was received for this work from any of the following organizations: National Institutes of Health (NIH); Wellcome Trust; Howard Hughes Medical Institute (HHMI); and other(s).

\section{Declarations}

Conflict of interest None of the authors has a financial interest in any of the products, devices or drugs mentioned in this manuscript.

Animal and human rights This article does not contain any studies with human participants or animals performed by any of the authors.

Informed consent For this type of study, formal consent is not required.

Open Access This article is licensed under a Creative Commons Attribution 4.0 International License, which permits use, sharing, adaptation, distribution and reproduction in any medium or format, as long as you give appropriate credit to the original author(s) and the source, provide a link to the Creative Commons licence, and indicate if changes were made. The images or other third party material in this article are included in the article's Creative Commons licence, unless indicated otherwise in a credit line to the material. If material is not included in the article's Creative Commons licence and your intended use is not permitted by statutory regulation or exceeds the permitted use, you will need to obtain permission directly from the copyright holder. To view a copy of this licence, visit http://creativecommons. org/licenses/by/4.0/.

\section{References}

1. Shahrabi-Farahani S et al (2014) Granulomatous foreign body reaction to dermal cosmetic fillers with intraoral migration. Oral Surg Oral Med Oral Pathol Oral Radiol 117(1):105-110

2. Klein AW (2006) Techniques for soft tissue augmentation: an "a to z." Am J Clin Dermatol 7(2):107-120

3. PlasticSurgery.org (2019) American society of plastic surgeons online resources. https://www.plasticsurgery.org/cosmeticprocedures/dermal-fillers. Accessed 27 Nov 2019

4. Broder KW, Cohen SR (2006) An overview of permanent and semipermanent fillers. Plast Reconstr Surg 118(3 Suppl):7S-14S

5. Owosho AA et al (2014) Orofacial dermal fillers: foreign body reactions, histopathologic features, and spectrometric studies. Oral Surg Oral Med Oral Pathol Oral Radiol 117(5):617-625

6. Christensen L (2007) Normal and pathologic tissue reactions to soft tissue gel fillers. Dermatol Surg 33(Suppl 2):S168-S175

7. Christensen L et al (2013) Bacterial infection as a likely cause of adverse reactions to polyacrylamide hydrogel fillers in cosmetic surgery. Clin Infect Dis 56(10):1438-1444
8. Kadouch JA et al (2013) Delayed-onset complications of facial soft tissue augmentation with permanent fillers in 85 patients. Dermatol Surg 39(10):1474-1485

9. Kadouch JA et al (2014) Complications after facial injections with permanent fillers: important limitations and considerations of MRI evaluation. Aesthet Surg J 34(6):913-923

10. Kadouch JA et al (2015) Granulomatous foreign-body reactions to permanent fillers: detection of CD123+ plasmacytoid dendritic cells. Am J Dermatopathol 37(2):107-114

11. Micheels P (2001) Human anti-hyaluronic acid antibodies: is it possible? Dermatol Surg 27(2):185-191

12. de Melo Carpaneda E, Carpaneda CA (2012) Adverse results with PMMA fillers. Aesthetic Plast Surg 36(4):955-963

13. de Vries CG, Geertsma RE (2013) Clinical data on injectable tissue fillers: a review. Exp Rev Med Dev 10(6):835-853

14. Marusza W et al (2019) Treatment of late bacterial infections resulting from soft-tissue filler injections. Infect Drug Resist 12:469-480

15. Marusza W et al (2019) The impact of lifestyle upon the probability of late bacterial infection after soft-tissue filler augmentation. Infect Drug Resist 12:855-863

16. Christensen L, Breiting V, Vuust J et al (2006) Adverse reactions following injection with permanent facial filler, polyacrylamide hydrogel (Aquamid): causes and treatment. Eur J Plast Surg 28:464-471

17. De Boulle K (2010) Critical reflections on ArteFill, a permanent injectable product for soft tissue augmentation: mechanism of action and injection techniques, indications, and applications. Aesthetic Plast Surg 34(3):287-289

18. Rudolph CM et al (1999) Foreign body granulomas due to injectable aesthetic microimplants. Am J Surg Pathol 23(1):113-117

19. Wiest LG, Stolz W, Schroeder JA (2009) Electron microscopic documentation of late changes in permanent fillers and clinical management of granulomas in affected patients. Dermatol Surg 35(Suppl 2):1681-1688

20. Duranti $F$ et al (1998) Injectable hyaluronic acid gel for soft tissue augmentation. A clinical and histological study. Dermatol Surg 24(12):1317-1325

21. Vedamurthy M, Vedamurthy A (2008) Dermal fillers: tips to achieve successful outcomes. J Cutan Aesthet Surg 1(2):64-67

22. Lemperle G, Rullan PP, Gauthier-Hazan N (2006) Avoiding and treating dermal filler complications. Plast Reconstr Surg 118(3 Suppl):92S-107S

23. Marusza W et al (2012) Probable biofilm formation in the cheek as a complication of soft tissue filler resulting from improper endodontic treatment of tooth 16 . Int $\mathbf{J}$ Nanomedicine $7: 1441-1447$

24. Netsvyetayeva I et al (2018) Skin bacterial flora as a potential risk factor predisposing to late bacterial infection after cross-linked hyaluronic acid gel augmentation. Infect Drug Resist 11:213-222

25. Eversole R et al (2013) Lip augmentation dermal filler reactions, histopathologic features. Head Neck Pathol 7(3):241-249

26. Ficarra G, Mosqueda-Taylor A, Carlos R (2002) Silicone granuloma of the facial tissues: a report of seven cases. Oral Surg Oral Med Oral Pathol Oral Radiol Endod 94(1):65-73

27. Daley $T$ et al (2012) Oral lesions associated with injected hydroxyapatite cosmetic filler. Oral Surg Oral Med Oral Pathol Oral Radiol 114(1):107-111

28. Bjarnsholt $\mathrm{T}$ et al (2009) Detection of bacteria by fluorescence in situ hybridization in culture-negative soft tissue filler lesions. Dermatol Surg 35(Suppl 2):1620-1624

29. Moher D et al (2009) Preferred reporting items for systematic reviews and meta-analyses: the PRISMA statement. Ann Intern Med 151(4): 264-269 (W64) 
30. Oxford Centre for evidence-based medicine (2011) Levels of evidence. https://www.cebm.net/wp-content/uploads/2014/06/ CEBM-Levels-of-Evidence-2.1.pdf. Accessed 27 Nov 2019

31. Saththianathan M et al (2017) The role of bacterial biofilm in adverse soft-tissue filler reactions: a combined laboratory and clinical study. Plast Reconstr Surg 139(3):613-621

32. Christensen L et al (2005) Adverse reactions to injectable soft tissue permanent fillers. Aesthetic Plast Surg 29(1):34-48

33. Alijotas-Reig J, Garcia-Gimenez V, Vilardell-Tarres M (2012) Tacrolimus in the treatment of chronic and refractory late-onset immune-mediated adverse effects related to silicone injections. Dermatol Surg 38(1):38-47

34. El-Khalawany $M$ et al (2015) Dermal filler complications: a clinicopathologic study with a spectrum of histologic reaction patterns. Ann Diagn Pathol 19(1):10-15

35. Lombardi $\mathrm{T}$ et al (2004) Orofacial granulomas after injection of cosmetic fillers. Histopathologic and clinical study of 11 cases. J Oral Pathol Med 33(2):115-120

36. Ledon JA et al (2013) Inflammatory nodules following soft tissue filler use: a review of causative agents, pathology and treatment options. Am J Clin Dermatol 14(5):401-411

37. Alijotas-Reig $\mathbf{J}$ et al (2012) Autoimmune/inflammatory syndrome (ASIA) induced by biomaterials injection other than silicone medical grade. Lupus 21(12):1326-1334

38. Emer J, Waldorf H (2011) Injectable neurotoxins and fillers: there is no free lunch. Clin Dermatol 29(6):678-690

39. Christensen LH (2009) Host tissue interaction, fate, and risks of degradable and nondegradable gel fillers. Dermatol Surg 35(Suppl 2):1612-1619

40. Karim RB et al (2006) Complications of polyalkylimide $4 \%$ injections (Bio-Alcamid): a report of 18 cases. J Plast Reconstr Aesthet Surg 59(12):1409-1414

41. Schelke LW et al (2009) Complications after treatment with polyalkylimide. Dermatol Surg 35(Suppl 2):1625-1628

42. Hirsch RJ, Stier M (2008) Complications of soft tissue augmentation. J Drugs Dermatol 7(9):841-845

43. Bergeret-Galley C, Latouche X, Illouz YG (2001) The value of a new filler material in corrective and cosmetic surgery: DermaLive and DermaDeep. Aesthetic Plast Surg 25(4):249-255

44. Christen U, von Herrath MG (2005) Infections and autoimmunity-good or bad? J Immunol 174(12):7481-7486

45. Alhede $\mathrm{M}$ et al (2014) Bacterial biofilm formation and treatment in soft tissue fillers. Pathog Dis 70(3):339-346

46. Lemperle G, Nicolau P, Scheiermann N (2011) Is there any evidence for biofilms in dermal fillers? Plast Reconstr Surg $128(2): 84 \mathrm{e}-85 \mathrm{e}$

47. Alijotas-Reig J, Fernandez-Figueras MT, Puig L (2013) Lateonset inflammatory adverse reactions related to soft tissue filler injections. Clin Rev Allergy Immunol 45(1):97-108

48. Alijotas-Reig J, Fernandez-Figueras MT, Puig L (2013) Inflammatory, immune-mediated adverse reactions related to soft tissue dermal fillers. Semin Arthritis Rheum 43(2):241-258

49. Lemperle $G$ et al (2009) Foreign body granulomas after all injectable dermal fillers: part 1. Possible causes. Plast Reconstr Surg 123(6):1842-1863

50. Nicolau PJ (2007) Long-lasting and permanent fillers: biomaterial influence over host tissue response. Plast Reconstr Surg 119(7):2271-2286

51. Anderson JM, Rodriguez A, Chang DT (2008) Foreign body reaction to biomaterials. Semin Immunol 20(2):86-100

52. Lowe NJ, Maxwell CA, Patnaik R (2005) Adverse reactions to dermal fillers: review. Dermatol Surg 31(11 Pt 2):1616-1625

53. Mariani E et al (2019) Biomaterials: foreign bodies or tuners for the immune response? Int J Mol Sci 20(3):636

54. Orentreich DS (2000) Liquid injectable silicone: techniques for soft tissue augmentation. Clin Plast Surg 27(4):595-612
55. Rongioletti $\mathrm{F}$ et al (2003) Granulomatous reaction after intradermal injections of hyaluronic acid gel. Arch Dermatol 139(6):815-816

56. Morhenn VB, Lemperle G, Gallo RL (2002) Phagocytosis of different particulate dermal filler substances by human macrophages and skin cells. Dermatol Surg 28(6):484-490

57. Lemperle $\mathrm{G}$ et al (2004) Migration studies and histology of injectable microspheres of different sizes in mice. Plast Reconstr Surg 113(5):1380-1390

58. Tomazic-Jezic VJ, Merritt K, Umbreit TH (2001) Significance of the type and the size of biomaterial particles on phagocytosis and tissue distribution. J Biomed Mater Res 55(4):523-529

59. Alijotas-Reig J, Garcia-Gimenez V, Vilardell-Tarres M (2009) Late-onset immune-mediated adverse effects after poly-L-lactic acid injection in non-HIV patients: clinical findings and longterm follow-up. Dermatology 219(4):303-308

60. DeLorenzi C (2013) Complications of injectable fillers, part I. Aesthet Surg J 33(4):561-575

61. Lowe NJ et al (2001) Hyaluronic acid skin fillers: adverse reactions and skin testing. J Am Acad Dermatol 45(6):930-933

62. Garcia-Domingo MI et al (2000) Disseminated and recurrent sarcoid-like granulomatous panniculitis due to bovine collagen injection. J Investig Allergol Clin Immunol 10(2):107-109

63. Israeli $\mathrm{E}$ et al (2009) Adjuvants and autoimmunity. Lupus 18(13):1217-1225

64. Schijns VE (2000) Immunological concepts of vaccine adjuvant activity. Curr Opin Immunol 12(4):456-463

65. Miro-Mur F et al (2009) Medical-grade silicone induces release of proinflammatory cytokines in peripheral blood mononuclear cells without activating T cells. J Biomed Mater Res B Appl Biomater 90(2):510-520

66. Jara LJ et al (2012) Still's disease, lupus-like syndrome, and silicone breast implants. A case of "ASIA" (Shoenfeld's syndrome). Lupus 21(2):140-145

67. Vera-Lastra O et al (2013) Autoimmune/inflammatory syndrome induced by adjuvants (Shoenfeld's syndrome): clinical and immunological spectrum. Exp Rev Clin Immunol 9(4):361-373

68. Shoenfeld Y, Agmon-Levin N (2011) 'ASIA'-autoimmune/inflammatory syndrome induced by adjuvants. J Autoimmun 36(1):4-8

69. Alijotas-Reig J et al (2009) Delayed immune-mediated adverse effects related to polyacrylamide dermal fillers: clinical findings, management, and follow-up. Dermatol Surg 35(Suppl 1):360-366

70. Alijotas-Reig J et al (2008) Delayed immune-mediated adverse effects of polyalkylimide dermal fillers: clinical findings and long-term follow-up. Arch Dermatol 144(5):637-642

71. Alijotas-Reig J, Garcia-Gimenez V (2008) Delayed immunemediated adverse effects related to hyaluronic acid and acrylic hydrogel dermal fillers: clinical findings, long-term follow-up and review of the literature. J Eur Acad Dermatol Venereol 22(2):150-161

72. Alijotas-Reig J (2009) Recurrent urticarial vasculitis related to nonanimal hyaluronic acid skin filler injection. Dermatol Surg 35(Suppl 1): 395-397 (discussion 397-398)

73. Pennington CA, Park JM (2015) Sublingual tacrolimus as an alternative to oral administration for solid organ transplant recipients. Am J Health Syst Pharm 72(4):277-284

74. Bass LS (2015) Injectable filler techniques for facial rejuvenation, volumization, and augmentation. Facial Plast Surg Clin North Am 23(4):479-488

Publisher's Note Springer Nature remains neutral with regard to jurisdictional claims in published maps and institutional affiliations. 